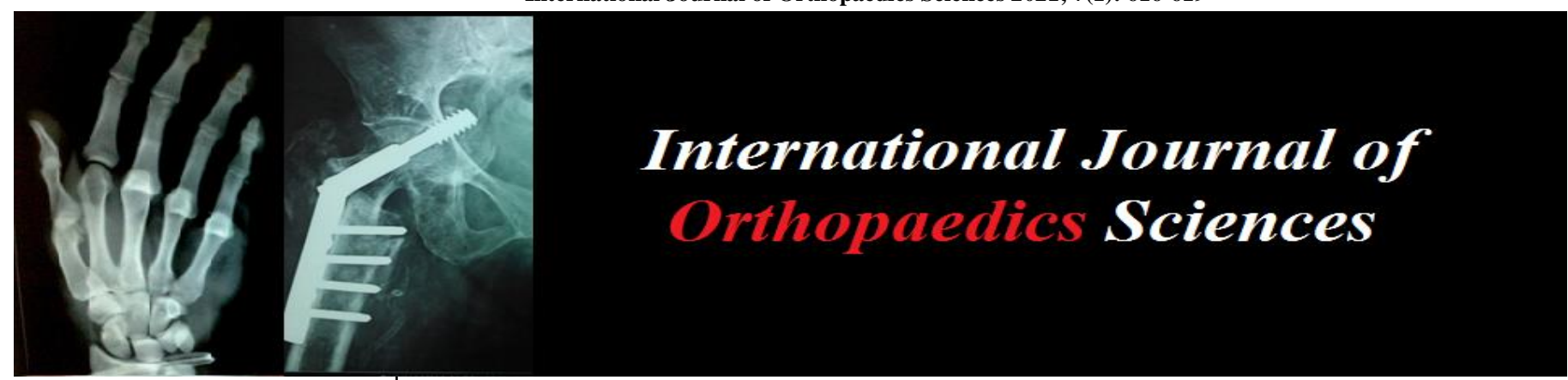

E-ISSN: 2395-1958

P-ISSN: 2706-6630

IJOS 2021; 7(1): 616-619

(C) 2021 IJOS

www.orthopaper.com

Received: 22-11-2020

Accepted: 24-12-2020

Uma Maheswara Reddy Surendra Multi Specialty Hospital, Tirupati, Andhra Pradesh, India
Corresponding Author: Uma Maheswara Reddy Surendra Multi Specialty Hospital, Tirupati, Andhra Pradesh, India

\section{A study of cocktail injection: Pain control and knee motion recovery after total knee replacement}

\section{Uma Maheswara Reddy}

DOI: https://doi.org/10.22271/ortho.2021.v7.i1j.2550

\section{Abstract}

Pain control after total knee replacement (TKR) is pivotal in postoperative rehabilitation. Usage of epidural analgesia or parenteral opioids can cause undesirable side effects hampering early recovery and rehabilitation. These side effects can be avoided by infiltration of an analgesic cocktail locally. Our study was performed to evaluate the benefits of a particular cocktail combination in patients undergoing TKR with respect to pain and knee motion recovery. Fifty consecutive patients who underwent simultaneous bilateral TKR were enrolled and received an intraoperative periarticular cocktail injection in the right knee (intervention) and normal saline in the left knee (control). Postoperative pain was recorded using the visual analog scale for each knee, and the time taken to achieve 90 degree of knee flexion was noted for each side. The cocktail injected knee had significantly less pain when compared with the control knee during the first 2 days and significantly shorter period to achieve 90 degree of knee flexion. The use of intraoperative periarticular cocktail injection significantly reduces early postoperative pain and provides better early knee motion.

Keywords: Total knee replacement, knee injury, intraarticular injection, pain, range of motion

\section{Introduction}

In patients with advanced knee arthritis, total knee replacement (TKR) has been found to be the most successful surgical procedure. However, early postoperative pain control is pivotal in reducing the hospital stay, increasing patient satisfaction, and for better rehabilitation. It also reduces the potential for postoperative complications such as pneumonia or deep vein thrombosis ${ }^{[1-4]}$. Severe postoperative pain is experienced in approximately $60 \%$ of the patients and moderate pain in approximately $30 \%$ of patients undergoing TKR ${ }^{[5-8]}$. Control of pain is achievable through multiple ways, and each has its own risks and benefits ${ }^{[9-12]}$. Epidural anesthesia is a common modality for providing effective pain relief during the postoperative period, but it hinders early mobilization and leads to complications such as hypotension, postoperative headache, and spinal infection ${ }^{[13-15]}$. Regional nerve blocks pose the risk of injuring neurovascular structures, hematoma formation, and infection ${ }^{[16,17]}$. Systemic opioids such as morphine or fentanyl can cause nausea, vomiting, drowsiness, respiratory depression, urinary retention, and constipation ${ }^{[18]}$. An innovative approach to pain management is to aim at controlling local pain pathways and receptors within the knee ${ }^{[19]}$. This has been possible through local intraarticular or periarticular injection of analgesic combinations which has good efficacy, is cost-effective, and is easy to administer without causing motor blockade. Also, it does not require any special technical skill for administration ${ }^{[5]}$. Various studies about periarticular injection have reported promising results from various combinations of drugs such as ketorolac, ropivacaine, bupivacaine, morphine sulfate, epimorphine, methylprednisolone, cefuroxime, epinephrine, and normal saline ${ }^{[20]}$. The patients experienced a prolonged narcotic-free postoperative period and also a reduced parenteral analgesia postoperatively ${ }^{[21]}$.

\section{Materials and Methods}

We included patients who underwent simultaneous bilateral TKR from Jan 2016 to Jul 2018 in our organization. For uniformity, we included only the patients for whom spinal anesthesia was the mode of anesthesia. 
Fifty consecutive patients who satisfied the inclusion criteria were selected for the study. All the patients had a full understanding of the 10-point visual analog pain scale (VAS). Exclusion criteria were patients with a history of allergy to the medications used in this study, abnormal renal or liver function, uncontrolled diabetes, and those who could not receive spinal anesthesia.

\subsection{Design of the study}

Our study was a prospective, double-blinded, placebo controlled trial. All included patients signed an informed consent form, and the methods of this trial were approved by the institutional ethics committee of our institute.

\subsection{Intervention}

For all the patients, intraoperative periarticular cocktail injection was given to the right knee and the left knee was the control that received a same volume of normal saline (110 $\mathrm{mL}$ ). The patients were blinded about which knee received the cocktail injection. All the patients received spinal anesthesia with a combination of $0.5 \%$ bupivacaine and $0.5 \mathrm{~mL}(25 \mathrm{mg})$ fentanyl. The antibiotic prophylaxis given was $1.5 \mathrm{~g}$ of injection cefuroxime 30 to 40 minutes before incision.

All the operations and the cocktail injections were performed by a single surgeon (first author) using a medial parapatellar arthrotomy approach. A periarticular cocktail injection consisting of $90 \mathrm{~mL}$ of normal saline, $17.5 \mathrm{~mL}$ of $5 \%$ bupivacaine, $2 \mathrm{~mL}$ of inj. ketorolac $(30 \mathrm{mg})$, and $0.5 \mathrm{~mL}$ of adrenaline (total volume: $110 \mathrm{~mL}$ ) was given to the right knee of all the patients involved in the study. The infiltration was performed using a 21-gauge needle and syringe. The aforementioned cocktail injection was formulated by the orthopaedic surgeon based on his or her clinical experience and past clinical studies.

The cocktail was injected at the following 7 anatomical zones ${ }^{[14]}$ as depicted in Figures 1:

Zone 1: medial retinaculum

Zone 2: medial collateral ligament and medial meniscus capsular attachment

Zone 3: posterior capsule

Zone 4: lateral collateral ligament and lateral meniscus capsular attachment

Zone 5: lateral retinaculum

Zone 6: patellar tendon and fat pad

Zone 7: cut ends of quadriceps muscle and tendon

Injection at zones 2, 3, and 4 were administered after making the tibial and femoral cuts and ligament balancing. At zones $1,5,6$, and 7 , the injection was administered after implant placement. Cemented cruciate-sacrificing implants were used for all the cases. After component placement and cement setting, tourniquet was released, and hemostasis was achieved before the wound was closed. No drains were used.

During the postoperative period, systemic analgesics used were intravenous injection of diclofenac $(75 \mathrm{mg}$ ) and inj. tramadol (100 $\mathrm{mg}$ ) along with inj. ondansetron $(4 \mathrm{mg})$ every 12 hours for the first 2 days followed by tablet naproxen 500 $\mathrm{mg}$ and tablet tramadol hydrochloride $(37.5 \mathrm{mg})$ with paracetamol $(325 \mathrm{mg}$ ) for the next 10 days. Buprenorphine patch $(10 \mathrm{mg})$ or oral pregabalin $(75 \mathrm{mg})$ were used in patients for whom the aforementioned medications were insufficient in controlling pain or could not be tolerated.

Apart from mechanical deep vein thrombosis (DVT) prophylaxis such as DVT stockings, inj. fondaparinux $2.5 \mathrm{mg}$ on the first day followed by oral aspirin $150 \mathrm{mg}$ daily for 6 weeks were given. Patients were mobilized using a walker after 3 to 4 hours of surgery on the same day, and range of motion (ROM) and isometric exercises were started. All the patients were observed till discharge and are being followed up regularly.

\subsection{Outcome from the measurement}

Postoperative pain over both the knees were separately recorded by the nurse, who was blinded about the study, using a 10 point VAS at 3,6,9,12,24 and 48 hours postoperatively, and then, at once-daily intervals till the fourth postoperative day. The VAS consists of a $10-\mathrm{cm}$ line, in which 0 indicates no pain and 10 indicates the worst imaginable pain ${ }^{[13-15]}$.

Postoperative range of active flexion was noted each day till the fourth postoperative day on both the knees separately by the physiotherapist, who was also blinded about the study.

Vitals monitoring included blood pressure, heart rate, and oxygen saturation. Any adverse reactions including allergic reactions, nausea, vomiting, urinary retention, or respiratory depression were also monitored till the patients were discharged.

\subsection{Statistical analysis}

The obtained data were tabulated, coded, and analyzed using SPSS, version 17, for Microsoft Windows. Descriptive statistics was reported as mean and standard deviation. Unpaired t test was used to test the statistical association between the intervention arm and control arm. For analyzing the change in pain scores over the same knee during the follow-up periods, we used repeated-measures analysis of variance. A post hoc test was conducted to assess the presence of any statistical significance between the 2 time points.

\section{Results and Discussions}

A total of 50 patients ( 50 pairs of knees) were included in the study. Osteoarthritis was the underlying condition in 47 patients, while the rest of them had rheumatoid arthritis.

The mean pain scores (VAS) at 3,6,9,12,24 and 48 hours, and at third and fourth days in both knees are enumerated in Table 1 and Figure 2. When compared with the control knee, a statistically significant reduction in pain score was noted in the cocktail injected knee at $63,6,9,12,24$ and 48 hours $(\mathrm{P}<$ .001 in all cases). However, the difference in the mean pain scores between both knees at the third (P 1/4 .684) and fourth (P 1/4 .251) days were not significant.

The mean time taken for achieving 90 degree flexion in the intervention and control knees were 1.70 and 2.82 days, respectively. The difference was found to be statistically significant $(\mathrm{P}<.001)$. Within the intervention group, there was a significant difference in the pain scores over different time points (Table 2). A post hoc analysis showed no significant difference within various time points on the first day $(3,6,9$, 12 and 24 hours) after surgery. However, a statistically significant difference in the pain scores was noted at 48 hours $(\mathrm{P}<.001), 72$ hours $(\mathrm{P}<.010)$, and 96 hours $(\mathrm{P}<.001)$, compared with the 24-hour score. Within the control group, there was a significant difference in pain scores over different time points (Table 2). However, a post hoc analysis showed that there was no significant difference within various time points on the first day $(3,6,9,12$ and 24 hours) after surgery, and statistically significant improvement was found only after 72 hours $(\mathrm{P}<.001)$ and 96 hours $(\mathrm{P}<.001)$, compared with the 24-hour value.

\subsection{Discussions}

During TKR, trauma to the tissues exaggerates the 
neurological responsiveness to pain by reducing the threshold of afferent nociceptive neurons and by central sensitization of excitatory neurons. This contributes to increased sensitivity to postoperative pain ${ }^{[11]}$. Hence, a multimodal approach for postoperative pain control has been particularly effective not only in relieving postoperative pain but also in facilitating earlier rehabilitation and improving postoperative ROM. It also reduces the complications of other modalities of pain management such as patient-controlled anesthesia (PCA), continuous epidural anesthesia, and femoral nerve block.

Table 1: VAS Comparisons between groups

\begin{tabular}{|c|c|c|c|c|c|c|}
\hline S. No & \begin{tabular}{|l|} 
Post-operative Duration \\
\end{tabular} & Group & Mean & Standard Deviation & Standard Error Mean & P Value \\
\hline \multirow{2}{*}{1} & \multirow{2}{*}{$3 \mathrm{~h}$} & Control & 3.75 & 1.939 & 0.194 & \multirow{2}{*}{$<.001 *$} \\
\hline & & Intervention & 1.99 & 1.419 & 0.142 & \\
\hline \multirow{2}{*}{2} & \multirow{2}{*}{$6 \mathrm{~h}$} & Control & 3.54 & 1.927 & 0.193 & \multirow{2}{*}{$<.001^{*}$} \\
\hline & & Intervention & 1.9 & 1.406 & 0.141 & \\
\hline \multirow{2}{*}{3} & \multirow{2}{*}{$9 \mathrm{~h}$} & Control & 3.34 & 1.778 & 0.178 & \multirow{2}{*}{$<.001^{*}$} \\
\hline & & Intervention & 1.87 & 1.376 & 0.138 & \\
\hline \multirow{2}{*}{4} & \multirow{2}{*}{$12 \mathrm{~h}$} & Control & 3.16 & 1.77 & 0.177 & \multirow{2}{*}{$<.001^{*}$} \\
\hline & & Intervention & 1.84 & 1.375 & 0.138 & \\
\hline \multirow{2}{*}{5} & \multirow{2}{*}{$24 \mathrm{~h}$} & Control & 2.64 & 1.365 & 0.137 & \multirow{2}{*}{$<.001^{*}$} \\
\hline & & Intervention & 1.6 & 0.656 & 0.066 & \\
\hline \multirow{2}{*}{6} & \multirow{2}{*}{$48 \mathrm{~h}$} & Control & 2.3 & 1.056 & 0.106 & \multirow{2}{*}{$<.001^{*}$} \\
\hline & & Intervention & 1.17 & 0.825 & 0.083 & \\
\hline \multirow{2}{*}{7} & \multirow{2}{*}{$3 d$} & Control & 1.2 & 1.056 & 0.106 & \multirow{2}{*}{0.685} \\
\hline & & Intervention & 1.18 & 1.036 & 0.104 & \\
\hline \multirow{2}{*}{8} & \multirow{2}{*}{$4 d$} & Control & 1.11 & 1.026 & 0.102 & \multirow{2}{*}{0.252} \\
\hline & & Intervention & 0.96 & 0.825 & 0.083 & \\
\hline
\end{tabular}

${ }^{*}$ Significant at $\mathrm{P}<.05$

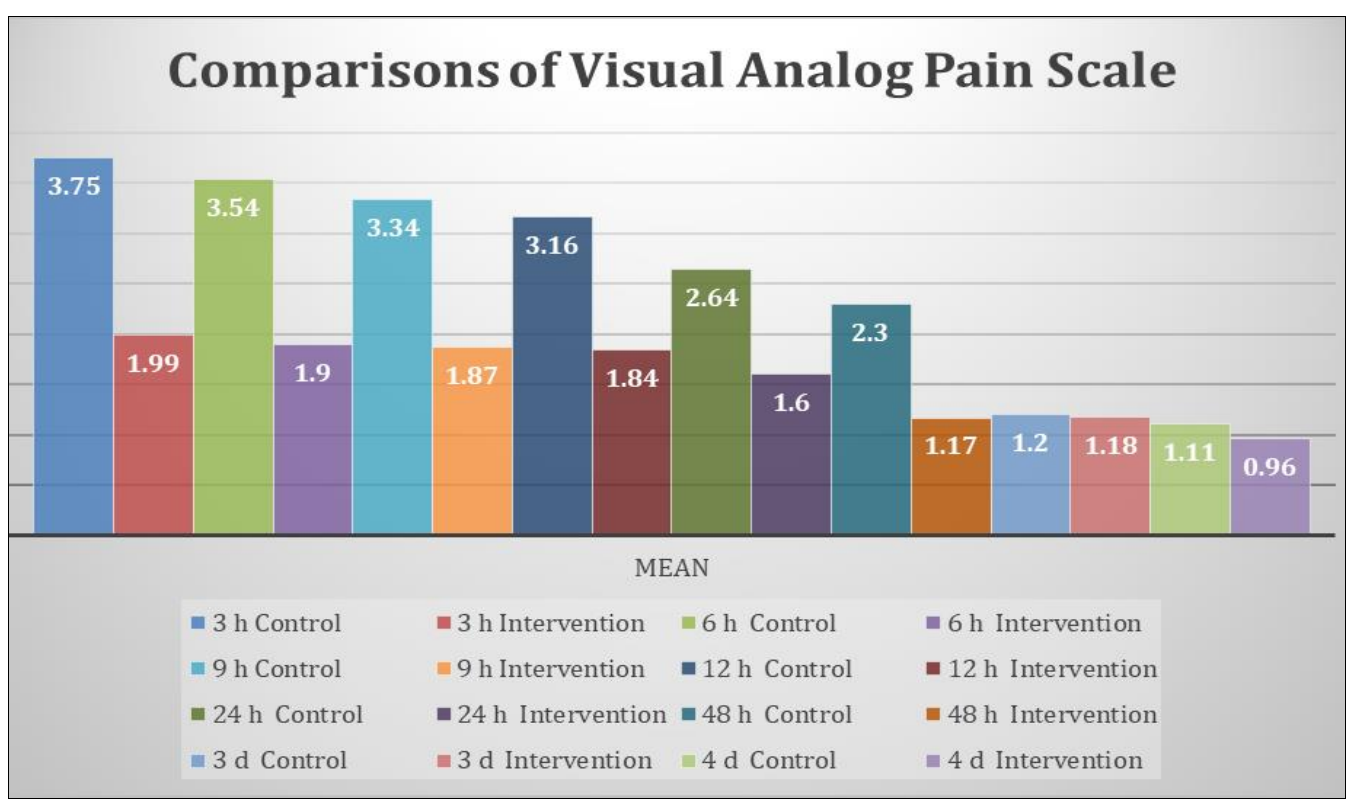

Fig 2: Comparisons of VAS

Table 2: Postoperative duration

\begin{tabular}{|c|c|c|c|c|c|}
\hline N= Sample Size & Group & Postoperative duration & Mean & Standard Deviation & P Value \\
\hline \multirow{16}{*}{200} & \multirow{8}{*}{ Control } & $3 \mathrm{~h}$ & 3.75 & 1.939 & \multirow{8}{*}{$<.001$} \\
\hline & & $6 \mathrm{~h}$ & 3.54 & 1.927 & \\
\hline & & $9 \mathrm{~h}$ & 3.34 & 1.778 & \\
\hline & & $12 \mathrm{~h}$ & 3.16 & 1.77 & \\
\hline & & $24 \mathrm{~h}$ & 2.64 & 1.365 & \\
\hline & & $48 \mathrm{~h}$ & 2.3 & 1.056 & \\
\hline & & $3 \mathrm{~d}$ & 1.2 & 1.056 & \\
\hline & & $4 \mathrm{~d}$ & 1.11 & 1.026 & \\
\hline & \multirow{8}{*}{ Intervention } & $3 \mathrm{~h}$ & 1.99 & 1.419 & \multirow{8}{*}{$<.001$} \\
\hline & & $6 \mathrm{~h}$ & 1.9 & 1.406 & \\
\hline & & $9 \mathrm{~h}$ & 1.87 & 1.376 & \\
\hline & & $12 \mathrm{~h}$ & 1.84 & 1.375 & \\
\hline & & $24 \mathrm{~h}$ & 1.6 & 0.656 & \\
\hline & & $48 \mathrm{~h}$ & 1.17 & 0.825 & \\
\hline & & $3 \mathrm{~d}$ & 1.18 & 1.036 & \\
\hline & & $4 \mathrm{~d}$ & 0.96 & 0.825 & \\
\hline
\end{tabular}


The cocktail injection was given in a periarticular manner. Significant reduction in pain (by VAS) was recorded over the knee where the injection was given (right side) compared with the opposite side at $3,6,9,12,24$, and 48 hours $(\mathrm{P}<.001)$. This is in comparison with the study by $\mathrm{Fu}$ et al. which showed VAS score at rest was significantly lower at 6, 10, 24, and 36 hours postoperatively in the trial group compared with the control group, although the difference was insignificant at 24 hours postoperatively, and at days 2, 7, and 15 between the 2 groups. VAS score during activity was also lower in the trial group at 24 and 36 hours postoperatively than that in the control group, although the difference was insignificant at days 2, 7, and $15^{[2,8]}$. Busch et al. noted that patients who received a periarticular intraoperative injection containing ropivacaine, ketorolac, epimorphine, and epinephrine used significantly less PCA during the first 24 hours postoperatively ${ }^{[1-4]}$. Nair et al also comparing the 2 groups of 40 knees each, reported that the cocktail injected patients reported significantly less PCA and postoperative pain recordings at $3,6,9,12,24,48$, and 72 hours after TKR.

\section{Conclusion}

In this study concludes that periarticular cocktail injection in TKR not only helps in relieving the pain but also aids in early recovery and rehabilitation. Because, a power analysis was not performed before commencing the study, and we just included patients belonging to a particular time frame. The optimal concentration of the individual components of the cocktail could not be determined, and further effort is required to comment on the superiority of 1 component over the other. Another question of debate is whether the infiltration of normal saline to the control side itself could incite pain mechanically even though we presumed normal saline by itself has no local pharmacological effects. Our study did not attempt at evaluating long-term clinical outcomes of the patients.

\section{Acknowledgements}

I acknowledge who supported the work and express my sincere thanks.

\section{References}

1. Nair, Vijayamohan, Radhamony Niranj, Rajendra Riju, Mishra Rahul. Effectiveness of intraoperative periarticular cocktail injection for pain control and knee motion recovery after total knee replacement. Arthroplasty Today 2019;15(3):320-324.

2. Fu P, Wu Y, Wu H, Li X, Qian Q, Zhu Y. Efficacy of intra-articular cocktail analgesic injection in total knee arthroplastyda randomized controlled trial. Knee 2009;16(4):280.

3. Fajardo M, Collins J, Landa J, Adler E, Meere P, Di Cesare PE. Effect of a perioperative intra-articular injection on pain control and early range of motion following bilateral TKA. Orthopedics 2011;34(5):e33.

4. Yuenyongviwat V, Pornrattanamaneewong C, Chinachoti $\mathrm{T}$, Chareancholvanich K. Periarticular injection with bupivacaine for postoperative pain control in total knee replacement: a prospective randomized double-blind controlled trial. Adv Orthop 2012, 107309.

5. Dalury DF. A state-of-the-art pain protocol for total knee replacement. Arthroplasty Today 2016;2(1):23.

6. Toftdahl K, Nikolajsen L, Haraldsted V, Madsen F, Tønnesen EK, Søballe K. Comparison of peri-and intraarticular analgesia with femoral nerve block after total knee arthroplasty: A randomized clinical trial. Acta Orthop 2007;78(2):172.

7. Vendittoli PA, Makinen P, Drolet $\mathrm{P}$ et al. A multimodal analgesia protocol for total knee arthroplasty: a randomized, controlled study. J Bone Joint Surg Am 2006;88(2):282.

8. Parvataneni HK, Shah VP, Howard H, Cole N, Ranawat AS, Ranawat CS. Controlling pain after total hip and knee arthroplasty using a multimodal protocol with local periarticular injections: a prospective randomized study. J Arthroplasty 2007;22(6):33.

9. Maheshwari AV, Blum YC, Shekhar L, Ranawat AS, Ranawat CS. Multimodal pain management after total hip and knee arthroplasty at the Ranawat Orthopaedic Center. Clin Orthop Relat Res 2009;467(6):1418.

10. Ranawat AS, Ranawat CS. Pain management and accelerated rehabilitation for total hip and total knee arthroplasty. J Arthroplasty 2007;22(7):12.

11. Busch CA, Shore BJ, Bhandari R et al. Efficacy of periarticular multimodal drug injection in total knee arthroplasty: a randomized trial. J Bone Joint Surg Am 2006;88(5):959.

12. Hernandez-Palazon J. Infiltration of the surgical wound with local anesthetic for postoperative analgesia in patients operated on for lumbar disc herniation. Comparative study of ropivacaine and bupivacaine. Rev Esp Anestesiol Reanim 2001;48(1):17.

13. Cherian MN, Mathews MP, Chandy MJ. Local wound infiltration with bupivacaine in lumbar laminectomy. Surg Neurol 1997;47(2):120.

14. Galindo RP, Marino J, Cushner FD, Scuderi GR. Periarticular regional analgesia in total knee arthroplasty: a review of the neuroanatomy and injection technique. Orthop Clin North Am 2015;46(1):1.

15. Badner NH, Bourne RB, Rorabeck CH, MacDonald SJ, Doyle JA. Intra-articular injection of bupivacaine in knee-replacement operations. Results of use for analgesia and for preemptive blockade. J Bone Joint Surg Am 1996;78(5):734.

16. $\mathrm{Ng} \mathrm{FY,} \mathrm{Ng} \mathrm{JK}$, Chiu KY, Yan $\mathrm{CH}$, Chan CW. Multimodal periarticular injection vs continuous femoral nerve block after total knee arthroplasty: A prospective, crossover, randomized clinical trial. J Arthroplasty 2012;27(6):1234.

17. Kelley TC, Adams MJ, Mulliken BD, Dalury DF. Efficacy of multimodal perioperative analgesia protocol with periarticular medication injection in total knee arthroplasty: A randomized, double-blinded study. J Arthroplasty 2013;28(8):1274.

18. Ritter MA, Koehler M, Keating EM, Faris PM, Meding JB. Intra-articular morphine and/or bupivacaine after total knee replacement. J Bone Joint Surg Br 1999;81(2):301.

19. Christensen CP, Jacobs CA, Jennings HR. Effect of periarticular corticosteroid injections during total knee arthroplasty: A double-blind randomized trial. J Bone Joint Surg Am 2009;91(11):2550.

20. Sauerland S, Nagelschmidt M, Mallmann P, Neugebauer EA. Risks and benefits of preoperative high dose methylprednisolone in surgical patients. Drug Saf 2000;23(5):449.

21. Gilron I. Corticosteroids in postoperative pain management: future research directions for a multifaceted therapy. Acta Anaesthesiol Scand 2004;48(10):1221. 